\title{
PERMANENT GROUPS. II
}

\author{
LEROY B. BEASLEY AND LARRY CUMMINGS ${ }^{1}$
}

\begin{abstract}
A permanent group is a group of nonsingular matrices on which the permanent function is multiplicative. We consider only permanent groups which contain the group of nonsingular diagonal matrices. If the underlying field is infinite of characteristic zero or greater than $n$, then each such permanent group consists only of matrices in which exactly one diagonal has all nonzero entries.
\end{abstract}

A permanent group is a group of nonsingular matrices on which the permanent function is multiplicative. One example is $\mathscr{D}_{n}(F)$, the group of nonsingular $n \times n$ diagonal matrices over the field $F$. Marcus and Minc [3] conjectured that $\Delta_{n}$, the groups of $n \times n$ matrices of the form $P D$ where $P$ is a permutation matrix and $D \in \mathscr{D}_{n}(F)$ is a maximal permanent group. In this conjecture the field $F$ was not specified and the first author [1] verified the conjecture for the field of complex numbers. In this paper we consider the set $\mathscr{P}_{n}(F)$ of those permanent groups which contain $\mathscr{D}_{n}(F)$, and characterize $\mathscr{P}_{n}(F)$ when $F$ is an infinite field with char $F=0$ or $>n$.

In [2] we defined the set $\mathscr{A}_{n}(F)$ of all $n \times n$ matrix groups $G$ of nonsingular matrices over $F$ satisfying:

(i) $\mathscr{D}_{n}(F) \leqq G$, and

(ii) $A, B \in G$ implies $A \circ B^{\mathrm{T}} \in \mathscr{D}_{n}(F)$

where "o" denotes the Hadamard product. Here, we are also concerned with the set $\mathscr{C}_{n}(F)$ of $n \times n$ nonsingular matrix groups over $F$ such that $G=H \cdot K=\{h k, h \in H, h \in K\}$ where:

(i) $H \in \mathscr{A}_{n}(F)$,

(ii) $K$ is a group of $n \times n$ permutation matrices, and

(iii) $\left\langle P H P^{-1} \mid P \in K\right\rangle \in \mathscr{A}_{n}(F)$.

Note that $\mathscr{D}_{n}(F) \leqq H$ for every $H \in \mathscr{A}_{n}(F)$ so that $\mathscr{D}_{n}(F) \leqq G$ for every $G \in \mathscr{C}_{n}(F)$.

Presented to the Society, August 28, 1972; received by the editors July 31, 1972. AMS (MOS) subject classifications (1970). Primary 15A15; Secondary $20 \mathrm{H} 20$.

Key words and phrases. Permanent, matrix group.

${ }^{1}$ The work of the second author was supported in part by grant A5284 of the National Research Council of Canada. 
Yet another set of matrix groups is $\mathscr{B}_{n}(F)$, consisting of all groups of $n \times n$ nonsingular matrices over $F$ such that:

(i) $\mathscr{D}_{n}(F) \leqq G$, and

(ii) $A \in G$ implies $A$ has a unique nonzero diagonal where, by an abuse of language, nonzero diagonal shall mean every entry of the diagonal is nonzero.

If $A$ is any $n \times n$ matrix with at least one nonzero diagonal we define $P_{A}\left(Q_{A}\right)$ to be any one of the permutation matrices such that $P_{A} A\left(A Q_{A}\right)$ has nonzero main diagonal. The number of nonzero entries in row $i$ (column $j$ ) of a matrix $B$ will be denoted by $r_{i}(B)\left(c_{j}(B)\right)$. The $(i, j)$ entry of the product of several matrices $A, B, C$ will be denoted $(A B C)_{i j}$. The set $\{1, \cdots, n\}$ will be denoted by $[n]$ and throughout $n>1$.

THEOREM. If $F$ is an infinite field and char $F=0$ or $>n$ then $\mathscr{P}_{n}(F)=$ $\mathscr{B}_{n}(F)=\mathscr{C}_{n}(F)$.

We demonstrate the inclusions $\mathscr{C}_{n}(F) \subseteq \mathscr{P}_{n}(F), \mathscr{P}_{n}(F) \subseteq \mathscr{B}_{n}(F)$, and $\mathscr{B}_{n}(F) \subseteq \mathscr{C}_{n}(F)$ in Propositions 1,3 , and 6 respectively with varying restrictions on $F$. The hypothesis of our theorem implies the hypotheses in each of these propositions.

1. Proposition. If $F$ is a field with at least 3 elements then $\mathscr{C}_{n}(F) \subseteq$ $\mathscr{P}_{n}(F)$.

Proof. If $A, B \in G=H \cdot K \in \mathscr{C}_{n}(F)$ let

$$
A=A_{1} P_{1} A_{2} P_{2} \cdots A_{s} P_{s} \text { and } B=B_{1} Q_{1} B_{2} Q_{2} \cdots B_{t} Q_{t}
$$

where $A_{i} \in H, P_{i} \in K$ for $i \in[s]$ and $B_{j} \in H, Q_{j} \in K$ for $j \in[t]$. Define the products

$$
\begin{aligned}
& \hat{P}_{i}=P_{i} P_{i+1} \cdots P_{s}, \quad i \in[s], \\
& \hat{Q}_{j}=Q_{1} Q_{2} \cdots Q_{j-1}, \quad j \in[t] \backslash\{1\}, \\
& \hat{Q}_{1}=I_{n} .
\end{aligned}
$$

Then

$$
\begin{aligned}
& A=A_{1} \hat{P}_{1} \hat{P}_{2}^{-1} A_{2} \hat{P}_{2} \hat{P}_{3}^{-1} A_{3} \cdots A_{s-1} \hat{P}_{s-1} \hat{P}_{s}^{-1} A_{s} \hat{P}_{s}, \\
& B=\hat{Q}_{1} B_{1} \hat{Q}_{1}^{-1} \hat{Q}_{2} B_{2} \hat{Q}_{2}^{-1} \cdots B_{t-1} \hat{Q}_{t-1}^{-1} \hat{Q}_{t} B_{t} Q_{t} .
\end{aligned}
$$

It is immediate that $\hat{P}_{1}^{-1} A \in\left\langle P H P^{-1} \mid P \in K\right\rangle$ and $B Q_{t}^{-1} \hat{Q}_{t}^{-1} \in\left\langle P H P^{-1} \mid P \in K\right\rangle$ while $\left\langle P H P^{-1} \mid P \in K\right\rangle \in \mathscr{A}_{n}(F)$.

Since $F$ has at least 3 elements, $\mathscr{A}_{n}(F) \subseteq \mathscr{P}_{n}(F)$ [2, Theorem 3.1]. Therefore,

$$
\text { per } \begin{aligned}
A B & =\operatorname{per}\left(\hat{P}_{1}^{-1} A B Q_{t}^{-1} \hat{Q}_{t}^{-1}\right) \\
& =\operatorname{per}\left(\hat{P}_{1}^{-1} A\right) \operatorname{per}\left(B Q_{t}^{-1} \hat{Q}_{t}^{-1}\right)=\operatorname{per} A \operatorname{per} B
\end{aligned}
$$


2. Lemma. Let $F$ be an infinite field and $A=\left(a_{i j}\right), B=\left(b_{i j}\right)$ be $n \times n$ matrices over $F$. If $J \subseteq[n] \times[n]$ and $(i, j) \in J$ always implies there exists $k \in[n]$ such that $a_{i k} b_{k j} \neq 0$ then there exists $D \in \mathscr{D}_{n}(F)$ for which $(A D B)_{i j} \neq 0$ for each $(i, j) \in J$.

Proof. Let $D=\operatorname{diag}\left[x_{1}, \cdots, x_{n}\right]$ be a diagonal matrix of indeterminates $x_{i}, i \in[n]$, over $F$ and consider the set of polynomials

$$
f_{i j}\left(x_{1}, \cdots, x_{n}\right)=(A D B)_{i j}=\sum_{s=1}^{n} a_{i s} x_{s} b_{s j} \quad(i, j) \in J .
$$

By hypothesis there is $k \in[n]$ such that $a_{i k} b_{k j} \neq 0$ so that each $f_{i j}$ is nonzero. Hence $f=\prod_{(i, j) \in J} f_{i j}$ is a nonzero polynomial. Since $F$ is infinite there is an $n$-tuple $\alpha=\left(\alpha_{1}, \cdots, \alpha_{n}\right)$ of elements from $F$ such that $f(\alpha) \neq 0[4$, Theorem 14, p. 38]. If, say, $\alpha_{i}=0$ let

$$
g(x)=f\left(\alpha_{1}, \cdots, \alpha_{i-1}, x, \alpha_{i+1}, \cdots, \alpha_{n}\right) .
$$

Because $F$ is infinite there is a nonzero $\beta$ in $F$ such that $g(\beta) \neq 0$. Accordingly we may assume $\alpha_{i} \neq 0$ for all $i \in[n]$ and choose $D=\operatorname{diag}\left[\alpha_{1}, \cdots, \alpha_{n}\right]$.

3. Proposition. If $F$ is an infinite field and char $F=0$ or $>n$ then $\mathscr{P}_{n}(F) \subseteq \mathscr{B}_{n}(F)$.

Proof. Suppose a group $G \in \mathscr{P}_{n}(F)$ contains a matrix $A$ with at least 2 distinct nonzero diagonals determined by the permutations $\sigma$ and $\tau$. Consider an $i \in[n]$ such that $\sigma(i) \neq \tau(i)$ and $C \in G$ such that $r_{i}(C)=\max \left\{r_{i}(B) \mid B \in G\right\}$. Since $A \in G$ and $r_{i}(A) \geqq 2, r_{i}(C) \geqq 2$. If $i \neq 1$ choose a permutation matrix $P$ interchanging 1 and $i$. Then $P G P^{-1} \in \mathscr{P}_{n}(F)$, $r_{1}\left(P C P^{-1}\right) \geqq r_{1}\left(P B P^{-1}\right)$ for all $B \in G$, and $r_{1}\left(P C P^{-1}\right) \geqq 2$ since $r_{1}\left(P A P^{-1}\right)=$ $r_{i}(A) \geqq 2$. Thus, without loss of generality we may take $i=1$.

Let $C=\left(c_{i j}\right), C^{-1}=\left(c_{i j}^{\prime}\right)$, and $\pi$ be a permutation which corresponds to a nonzero diagonal of $C^{-1}$; i.e., $c_{i \pi^{-1}(i)}^{\prime} \neq 0$ for all $i \in[n]$. In Lemma 2 take $A=C, B=C^{-1}$, and

$$
J=\{(i, i) \mid i \in[n]\} \cup\left\{\left(1, \pi^{-1}(t)\right) \mid C_{1 t} \neq 0\right\} .
$$

Lemma 2 then yields $D \in \mathscr{D}_{n}(F)$ such that $E=C D C^{-1}$ has nonzero main diagonal and $r_{1}(E)=r_{1}(C)$, since for all $i \in[n]$ there exists $k \in[n]$ such that $c_{i k} c_{k i}^{\prime} \neq 0$ (because $\sum_{k=1}^{n} c_{i k} c_{k i}^{\prime}=1$ ) and since for $t$ such that $c_{1 t} \neq 0$ we have $c_{1 t} c_{t \pi^{-1}(t)}^{\prime} \neq 0$.

Let $E=\left(e_{i j}\right)$. In particular $e_{11} \neq 0$ so we may assume $e_{1 j} \neq 0$ for $j \in[m]$ and $e_{1 j}=0$ for $j \in[n] \backslash[m]$ since, if not, there exists a permutation matrix $P$ fixing row 1 and column 1 in $P E P^{-1}$ and which gives $P E P^{-1}$ the desired form. This is possible because $P G P^{-1}$ is also in $\mathscr{P}_{n}(F)$. 
For arbitrary $B \in G, B Q_{B}=\left(b_{i j}^{\prime}\right)$ has the form

$$
\left(\begin{array}{ll}
B_{1} & 0 \\
B_{3} & B_{4}
\end{array}\right)
$$

where $B_{1}$ is $m \times m$ and the zero block may be empty; otherwise, if $b_{s t}^{\prime} \neq 0$ for some $s \in[m]$ and $t \in[n] \backslash[m]$ then Lemma 2 implies the existence of $D \in \mathscr{D}_{n}(D)$ such that $E D B Q_{B}$ has more than $m$ nonzero entries in row 1 , contradicting $r_{1}(E) \geqq r_{1}(B)$ for all $B \in G$. This follows upon taking $A=E$, $B=B Q_{B}=\left(b_{i j}^{\prime}\right)$, and $J=\{(1, i) \mid i \in[m] \cup\{t\}\}$ in Lemma 2. For, $e_{1 i} \cdot b_{i i}^{\prime} \neq 0$ when $i \in[m]$ and $B Q_{B}$ has nonzero main diagonal while $e_{i s} b_{s t}^{\prime} \neq 0$ since $s \in[m]$.

Now $A$ has 2 nonzero diagonals $\sigma$ and $\tau$ such that $\sigma(1) \neq \tau(1)$. There exists $i \neq 1$ such that $\sigma(1)=\tau(i)$; i.e., $a_{1 \sigma(1)} \neq 0$ and $a_{i \sigma(1)}=a_{i \tau(i)} \neq 0$. Choosing $Q_{A}$ so that $\left(A Q_{A}\right)_{11}=a_{1 \sigma(1)}$ and $\left(A Q_{A}\right)_{i 1}=a_{i \sigma(1)}$ we find that $\max \left\{c_{1}\left(B Q_{B}\right) \mid B \in G\right\} \geqq 2$ and so $r=\max \left\{c_{1}\left(B_{1}\right) \mid B \in G\right\} \geqq 2$ in $B Q_{B}=\left(\begin{array}{cc}B_{1} & 0 \\ B_{3} & B_{4}\end{array}\right)$.

Let $T \in G$ satisfy $c_{1}\left(T_{1}\right)=\max \left\{c_{1}\left(B_{1}\right) \mid B \in G\right\}$ where $T_{1}$ is the upper left-hand block in the decomposition (3.1) of $T$. By Lemma 2 there exists $D \in \mathscr{D}_{n}(F)$ such that $\left(T D T^{-1}\right)_{i j} \neq 0$ when $i=j \in[n]$ and $c_{1}\left(T D T^{-1}\right)=c_{1}\left(T_{1}\right)$. Let $F=T D T^{-1}$. In addition we may assume that $f_{j 1} \neq 0$ for $j \in[r]$ and $f_{j \mathbf{1}}=0$ for $j \in[m] \backslash[r]$ since, if not, there exists a permutation matrix $P$ which fixes rows and columns $1, m+1, \cdots, n$ and gives $P F P^{-1}$ the desired form. This is possible because $P G P^{-1}$ is also in $\mathscr{P}_{n}(F)$; and $P E P^{-1}$ has the same form as $E$ since $P$ does not permute columns $[n] \backslash[m]$.

An argument similar to the one showing that for arbitrary $B \in G$ the matrix $B Q_{B}$ has the form (3.1) shows that $P_{B} B$ has the further decomposition

$$
\left(\begin{array}{cc|c}
B_{11} & B_{12} & \\
0 & B_{13} & B_{2} \\
\hline B_{3} & B_{4}
\end{array}\right)
$$

where the zero block may be empty.

Another application of Lemma 2 yields $D \in \mathscr{D}_{n}(F)$ such that $K=F D E$ has nonzero main diagonal and the form

$$
\left(\begin{array}{cc:c}
K_{11} & K_{12} & 0 \\
0 & K_{13} & \\
\hdashline K_{2} & K_{3}
\end{array}\right)
$$

where $K_{11}$ is an $r \times r$ block with no zero entries and $r \geqq 2$. The existence of the zero blocks follows immediately from (3.1) and (3.2) since we can take $P_{K}=Q_{K}=I$. 
Letting $A=K_{11}$ and $B=K_{11}^{-1}$, Lemma 2 ensures the existence of $D_{11} \in$ $\mathscr{D}(F)$ such that $K_{11} D_{11} K_{11}^{-1}$ has all entries nonzero since $K_{11}$ does. Letting $D=\left(\begin{array}{cc}D_{11} & 0 \\ 0 & I\end{array}\right)$ we have that

$$
\mathscr{U}=K D K^{-1}\left(\begin{array}{cc:c}
K_{11} D_{11} K_{11}^{-1} & \mathscr{U}_{12} & 0 \\
0 & I & \\
\hdashline \mathscr{U}_{2} & & I
\end{array}\right)
$$

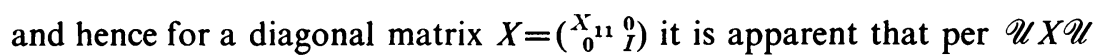
$=(\text { per } \mathscr{U})^{2}$ per $X$ if and only if $\operatorname{per}\left(\mathscr{U}_{11} X_{11} \mathscr{U}_{11}\right)=\left(\operatorname{per} \mathscr{U}_{11}\right)^{2}$ per $X_{11}$. If $X_{11}=\operatorname{diag}[x, 1, \cdots, 1]$ and $x$ is an indeterminate over $F$ then the coefficient of $x^{r}$ in the polynomial $k(x)=\operatorname{per} \mathscr{U}_{11} X_{11} \mathscr{U}_{11}-\left(\operatorname{per} \mathscr{U}_{11}\right)^{2} x$ is $r ! \prod_{i=1}^{r} U_{i 1} U_{1 \sigma(i)}$ which is nonzero for char $F=0$ or char $F>n \geqq r$. Since $F$ is an infinite field there is a nonzero $\alpha \in F$ such that $k(\alpha) \neq 0$. This contradicts the supposition that some $G \in \mathscr{P}_{n}(F)$ contains a matrix with more than one nonzero diagonal.

4. Lemma. Let $A=\left(a_{i j}\right), B_{1}=\left(b_{i j}\right), B_{1}^{\prime}=\left(b_{i j}^{\prime}\right)$ and $B_{2}=\left(c_{i j}\right)$ be $n \times n$ matrices over an infinite field $F$ satisfying:

(i) $A$ has at least one nonzero diagonal, and

(ii) $b_{i j} \neq 0$ implies $b_{i j}^{\prime} \neq 0, i, j \in[n]$.

There exist $D_{1}, D_{2} \in \mathscr{D}_{n}(F)$ such that $\left(B_{1} Q_{A}^{-1} B_{2}\right)_{i j} \neq 0$ implies

$$
\left(B_{1}^{\prime} D_{1} A D_{2} B_{2}\right)_{i j} \neq 0, \quad i, j \in[n] .
$$

Proof. Let $D_{1}=\operatorname{diag}\left[x_{1}, \cdots, x_{n}\right]$ and $D_{2}=\operatorname{diag}\left[y_{1}, \cdots, y_{n}\right]$ where the diagonal entries are indeterminates over $F$. Now,

$$
\left(B_{1} Q_{A}^{-1} B_{2}\right)_{i j}=\sum_{k=1}^{n} b_{i k} c_{\sigma^{-1}(k) j} \text { and }\left(B_{1}^{\prime} D_{1} A D_{2} B_{2}\right)_{i j}=\sum_{k=1}^{n} \sum_{t=1}^{n} b_{i k}^{\prime} x_{k} a_{k t} y_{t} c_{t j} \text {. }
$$

Consider the set $\mathscr{M}=\left\{\left(B_{1}^{\prime} D_{1} A D_{2} B_{2}\right)_{i j} \mid\left(B_{1} Q_{A}^{-1} B_{2}\right)_{i j} \neq 0\right\}$ of polynomials in the variables $x_{1}, \cdots, x_{n}, y_{1}, \cdots, y_{n}$. Each polynomial in $\mathscr{M}$ has the form

$$
b_{i s}^{\prime} x_{s} a_{s \sigma^{-1}(s)} y_{\sigma^{-1}(s)} c_{\sigma^{-1}(s) j}+f_{i j}\left(x_{1}, \cdots, x_{n}, y_{1}, \cdots, y_{n}\right)
$$

where the polynomial $f_{i j}$ has no terms in $x_{s} y_{\sigma^{-1(s)}}$ and $s$ may be chosen so that $b_{i s}^{\prime} a_{s \sigma^{-1}(s)} c_{\sigma^{-1}(s) j} \neq 0$. This is possible because $\left(B_{1} Q_{A}^{-1} B_{2}\right)_{i j}=$ $\sum_{k=1}^{n} b_{i k} c_{\sigma^{-1}(k) j} \neq 0$ so for some $s, b_{i s} c_{\sigma^{-1}(s) j} \neq 0$ and by (ii) we have $b_{i s}^{\prime} c_{\sigma^{-1}(s) j} \neq 0$ and also $0 \neq\left(A Q_{A}\right)_{s s}=a_{s \sigma^{-1}(s)}$, for $s \in[n]$.

Now, $\prod_{f \in, \|} f \neq 0$ since each $f \in \mathscr{M}$ is a nonzero polynomial. Hence by [4, Theorem 14, p. 38] there exists a $2 n$-tuple $\alpha=\left(\alpha_{1}, \cdots, \alpha_{2 n}\right)$ of elements in $F$ such that $\Pi_{f} . / I f(\alpha) \neq 0$ because $F$ is infinite and were some $\alpha_{i}=0$ we could find $\beta \neq 0$ in $F$ such that

$$
\prod_{f \in . / /} f\left(\alpha_{1}, \cdots, \alpha_{i-1}, \beta, \alpha_{i+1}, \cdots, \alpha_{2 n}\right) \neq 0 .
$$


Accordingly we may assume $\alpha_{i} \neq 0$ for all $i \in[2 n]$ and setting $D_{1}=$ $\operatorname{diag}\left[\alpha_{1}, \cdots, \alpha_{n}\right]$ and $D_{2}=\operatorname{diag}\left[\alpha_{n+1}, \cdots, \alpha_{2 n}\right]$ we obtain the conclusion.

5. Lemma. If $G$ is a maximal group in $\mathscr{B}_{n}(F)$ and $F$ is an infinite field then $Q_{A} \in G$.

Proof. Suppose $G$ contains $A$ with $Q_{A} \notin G$. Obviously $Q_{A}^{-1} \notin G$. The maximality of $G$ implies that $\left\langle G, Q_{A}^{-1}\right\rangle$ contains a matrix with at least two nonzero diagonals, say

$$
B_{1} Q_{A}^{\alpha_{1}} B_{2} Q_{A}^{\alpha_{2}} \cdots B_{m-1} Q_{A}^{\alpha_{m-1}} B_{m}
$$

where we may take each $\alpha_{i}=-1, i \in[m-1]$.

By Lemma 4 there are $D_{1}, D_{2} \in \mathscr{D}_{n}(F)$ such that $B_{1} D_{1} A D_{2} B_{2}$ has nonzero entries at least where $B_{1} Q_{A}^{-1} B_{2}$ does. Replacing $B_{1}$ by $B_{1} Q_{A}^{-1} B_{2}$ and $B_{1}^{\prime}$ by $B_{1} D_{1} A D_{2} B_{2}$ in Lemma 4 we see that $\left(B_{1} D_{1} A D_{2} B_{2}\right) \cdot D_{3} A D_{4} B_{3}$ has nonzero entries at least where $B_{1} Q_{A}^{-1} B_{2} Q_{A}^{-1} B_{3}$ does for some $D_{3}$, $D_{4} \in \mathscr{D}_{n}(F)$. Repeating this argument as necessary we obtain an element of $G$, namely $B_{1} D_{1} A D_{2} B_{2} \cdots D_{2 m-3} A D_{2 m-2} B_{m}$ which will have nonzero entries at least where (5.1) does and hence will have two distinct nonzero diagonals since (5.1) does.

\section{Proposition. If $F$ is an infinite field then $\mathscr{B}_{n}(F) \subseteq \mathscr{C}_{n}(F)$.}

Proof. Let $G$ be a maximal group in $\mathscr{B}_{n}(F)$. For very $A \in G$ there is exactly one permutation matrix $Q_{A}$ such that $A Q_{A}$ has nonzero main diagonal. Let $H=\left\langle A Q_{A} \mid A \in G\right\rangle$ and $K=\left\langle Q_{A} \mid A \in G\right\rangle$. By the previous lemma both $H$ and $K$ are subgroups of $G$ and therefore the complex $H \cdot K$ is contained in $G$. On the other hand each $A \in G$ may be written $A=A Q_{A} Q_{A}^{-1}$ which implies $G=H \cdot K$.

If $H \notin \mathscr{A}_{n}(F)$ there are $A, B \in H$ such that $a_{i j} b_{j i} \neq 0$ and $i \neq j$. Let $D_{j}=\operatorname{diag}[1, \cdots, x, \cdots, 1]$ where $x$ is diagonal entry $j$ and set $E=A D_{j} B$. Since $F$ has at least 3 nonzero elements, $x$ may be chosen so that if $E=\left(e_{i j}\right)$ then

$$
e_{i j}=a_{i j} x b_{j j}+\sum_{k \neq j} a_{i k} b_{k j} \neq 0 \text { and } e_{j i}=a_{j j} x b_{j i}+\sum_{k \neq j} a_{j k} b_{k i} \neq 0 .
$$

Since $H$ contains $\mathscr{D}_{n}(F)$ the matrix $E$ is in $H$ so has nonzero main diagonal entries. Therefore $E$ has at least 2 nonzero diagonal entries, contradicting $G \in \mathscr{B}_{n}(F)$. Hence $H \in \mathscr{A}_{n}(F)$.

If $A \in H$ then $A=B Q_{B}$ for some $B \in G$ and has a nonzero main diagonal. Thus any $P A P^{-1}$ with $P \in K$ has a nonzero main diagonal; i.e., $Q_{P^{\prime} \cdot l^{\prime, 1}}=I$ so that $P A P^{-1}=P A P^{-1} Q_{P^{\prime} A l^{-1}} \in H$. Thus $\left\langle P H P^{-1} \mid P \in K\right\rangle \in \mathscr{A}_{n}(F)$, completing the requirements for $G \in \mathscr{C}_{n}(F)$. 


\section{REFERENCES}

1. LeRoy B. Beasley, Maximal groups on which the permanent is multiplicative, Canad. J. Math. 21 (1969), 495-497; Corrigendum, ibid. 22 (1970), 192. MR 41 \#1757.

2. LeRoy B. Beasley and Larry Cummings, Permanent groups, Proc. Amer. Math. Soc. 34 (1972), 351-355.

3. M. Marcus and H. Minc, Permanents, Amer. Math. Monthly 72 (1965), 577-591. MR 31 \#1266.

4. O. Zariski and P. Samuel, Commutative algebra, Vol. I, University Series in Higher Math., Van Nostrand, Princeton, N.J., 1958. MR 19, 833.

Department of Pure Mathematics, University of Waterloo, Waterloo, Ontario, CANADA 